\title{
Précis of The Boundary Stones of Thought
}

\author{
Ian Rumfitt ${ }^{1}$
}

Published online: 23 May 2018

(C) Springer Science+Business Media B.V., part of Springer Nature 2018

If pressed, I could sum up The Boundary Stones of Thought in six words: classical logic good; classical semantics bad. However, somewhat less telegraphically...

The book aims to advance rational debate between adherents of rival logics of conjunction, disjunction, and negation. For the most part, the rivalry I focus on is classicists versus intuitionists (although classicists clash with quantum logicians in $\S 6.6)$. Debates over basic logical laws tend to fall prey to a vitiating circularity: in giving metalogical arguments for the soundness of their preferred system, adherents appeal to rules which their rivals do not accept. I aim to avoid this by finding semantic theories which are stable in the sense of delivering the same verdicts as to the validity of arguments even after switching from one set of metalogical rules to its rival. (Clearly, a stable theory cannot be homophonic.) The book interweaves assessments of various candidate semantic theories with analyses of particular arguments against classical laws, especially Excluded Middle and Double Negation Elimination. Even when those arguments cast serious doubt upon the Principle of Bivalence, I contend, they are ineffective against classical logic per se.

As a preliminary, Part One asks what logical laws are. Following Frege, I answer that they are 'laws of the laws of thought'. That is, they are higher-order principles which extend the scope of the various implicative relations that set the standards for correct deduction in rigorous special sciences such as geometry, mechanics, and electromagnetism. Thus the Law of Contraposition says that whenever $A$ stands to $B$ in an implicative relation $R$, any negation of $B$ stands in $R$ to any negation of $A$. An implicative relation is one which preserves actual truth and satisfies the 'Tarskian' structural conditions of Reflexivity, Monotonicity, and Cut. This account, I argue, offers the best explanation of the epistemic utility of logic.

Ian Rumfitt

ian.rumfitt@all-souls.ox.ac.uk

$1 \quad$ All Souls College, Oxford, UK 
By the Lindenbaum-Scott Theorem, each implicative relation is one which preserves truth at every member of an associated space of possibilities. My account of logical laws entails that there is a privileged relation of logical consequence which preserves truth at every logical possibility. Logical possibility emerges as the weakest species of possibility associated with an implicative relation. This yields a defence of Ian McFetridge's thesis that logical necessity is the strongest form of alethic necessity. I also explain where some purported counterarguments to McFetridge go wrong.

In Part Two, I turn in earnest to the quest for stable semantic theories. I consider the first candidate alongside the attack on classical logic in Michael Dummett's early paper 'Truth'. The kernel of that attack is an 'exclusionary' conception of content whereby what a statement says is given by the possibilities that it rules out. I develop this conception into an explicit semantic theory for sentential languages and show that, when the metalogic is classical, the classical rules for the connectives are sound and complete with respect to it. However, if the metalogic is intuitionistic, the semantics still validates the equivalence of any statement with its double negation, an equivalence which the intuitionist does not always accept. Thus intuitionistic logic does not cohere with the proposed semantics, and Dummett's argument for replacing classical with intuitionistic logic on the basis of the exclusionary conception fails.

Dummett later attacked classical logic from a different direction. He argued (a) that a statement's sense is best given by specifying the circumstances in which it would be verified, rather than the conditions under which it would be true; and (b) that the model for a verificationist semantic theory for a natural language should be Heyting's semantics for the language of intuitionistic mathematics. I give reasons for rejecting both (a) and (b). I further argue that, even if one accepts (a), one should reject (b). Heyting's theory limps even as an account of statements of constructive mathematics and is hopeless when applied to empirical statements. If one must be a verificationist, one does better to adopt the semantic theory I call 'evidentialism'. That theory, however, coheres with classical logic.

A less direct attack on classical logic is suggested by some writings by John McDowell. McDowell takes the senses of statements to be given by their truthconditions. Even given a classical metalogic, however, his account vindicates the logical truth of instances of Excluded Middle only if we have some logical guarantee of the truth of the Principle of Bivalence. McDowell thinks that we have no such guarantee, and hence no reason to be confident that classically valid arguments necessarily preserve truth.

In assessing this attack, we need an account of the 'conditions' under which a given statement would be true. In contrast to the prevailing account in terms of possible worlds, I take these to be the statement's truth-grounds-ways things might have been such that, had things been that way, they would have been as the statement in question (actually) says things are. Entities of this general kind I call possible states of affairs or possibilities; these differ from possible worlds in that a possibility need not be fully determinate. There is a natural operation of closure on the space of possibilities: $x$ belongs to the closure of a set $U$ if and only if $x$ includes what all the members of $U$ have in common. A set is called closed when it is 
identical with its own closure. I argue that the truth-grounds of any statement must form a closed set and propose semantic principles for conjunction and disjunction. The truth-grounds of $\lceil A$ and $B\rceil$ are the intersection of the truth-grounds of $A$ with the truth-grounds of $B$. The truth-grounds of $\lceil A$ or $B\rceil$ are the closure of the union of the truth-grounds of $A$ with the truth-grounds of $B$. Adherents of a wide range of logics will accept these principles.

There is also a natural notion of the combination of two possibilities. Closure induces a partial order $\leq$ on possibilities and the combination $x \bullet y$ is the $\leq$-least possibility $z$ such that $x \leq z$ and $y \leq z$. Where $x$ and $y$ are incompatible, $x \bullet y$ s taken to be an impossible state of affairs, $\perp$. This yields a semantic treatment of negation. The orthocomplement of $U, U^{\perp}$, contains exactly those states of affairs that are incompatible with all the members of $U$. The truth-grounds of $\lceil$ Not $A\rceil$ are the members of the orthocomplement of the truth-grounds of $A$.

This theory of truth-grounds is stable with respect to the dispute between classical and intuitionistic logicians; as stated, it validates intuitionistic logic whether the metalogic is classical or intuitionistic. In order to validate classical logic, we must add a further postulate about the space of possibilities-viz. every closed set of possibilities is the orthocomplement of another closed set. Given this postulate, all the classical logical laws are validated even if the metalogic is intuitionistic. I reconfigure some historic arguments for, or against, contested principles of classical logic as arguments for, or against, this further postulate. The reconfiguration shows why adherents of rival logics need not attach different senses to the connectives.

I then extend this approach to two particular areas where principles of classical logic have been thought to be inapplicable. Treating the logic of vague terms requires relativizing truth, not only to possibilities, but also to admissible determinations of such terms' senses. I advance a theory of this latter kind of relativization, not admittedly for all vague terms, but for the large and important sub-class of 'polar' predicates. Their admissible determinations, I contend, will be regular open sets in a topology induced by the poles by reference to which their meaning is given. The resulting semantics shows that there is no deviation from classical logic if we require that a valid argument preserves truth with respect to every admissible determination of sense. However, it allows that a disjunctive statement may be true even though none of its disjuncts is true. This last point, I argue, provides a solution to the Paradox of the Heap within classical logic.

Another area where classical rules have been doubted is transfinite set theory, at least under a non-Platonist conception of sets. Here, I start by assuming that the relevant space of possibilities comprises consistent extensions of $\mathrm{ZF}^{2}$, and argue that the appropriate closure operation is that of double orthocomplementation. While this vindicates classical logic, the analysis casts doubt on certain principles of $\mathrm{ZF}^{2}$, viz. the Power Set Axiom and the unrestricted schemata of Collection and Separation. The proper terminus for a non-Platonist account of set theory, I suggest, may not be $\mathrm{ZF}^{2}$ with an intuitionistic logic, but a weaker set theory, $\mathrm{KP} \omega$, with classical logic, at least for statements involving bounded quantification. (Whether unbounded quantification over sets makes sense is an interesting question I leave open.) 
The proposed treatments of vagueness and set theory require accepting all instances of Excluded Middle while allowing that some statements are neither true nor false. In the book's last chapter, I analyse the well-known deduction (the 'Simple Argument') which purports to derive the Principle of Bivalence, given a classical logic, from uncontentious premisses about truth and falsehood. When properly understood, I argue, those premisses are far from uncontentious, and in fact the analyses of vague terms and the language of set theory show that they are false in general. A classical logician, then, is not committed to Bivalence and should not accept it. I end by comparing Georg Kreisel's argument for the bivalence of the Continuum Hypothesis with the Simple Argument for the same conclusion. 